المنشآت المتميزة تراثياً وأثرياً وفنياً( قصير عمرة ودير القديس هيلاريون أنموذجاً)

Distinguished installations in The Heritage, Archeology and art (Qusayr Amra and Saint Hilarion Monastery as A model)

\author{
الباحثة/ منى سمير مقاط \\ بكالوريوس تاريخ وآثار - الجامعة الإسلامية بغزة \\ Researcher. Mona Samir Moqat
}

BA in History and Archeology - Islamic University of Gaza munasa1994@gmail.com

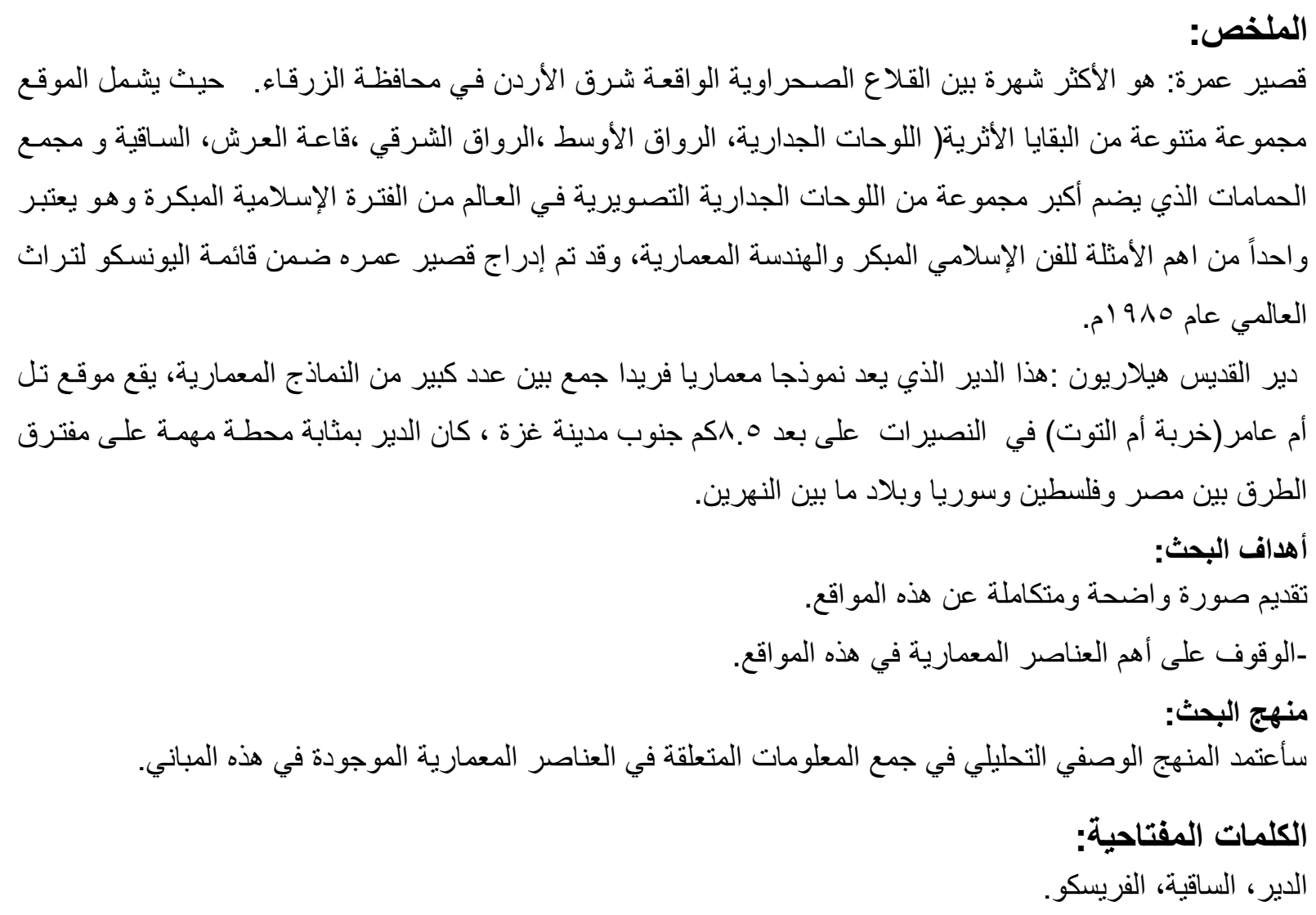

\title{
Abstract:
}

Qusayr Amra: It is the most famous of the desert castles located in eastern Jordan in the Governorate

Zarqa. Where the site included a variety of archaeological sites (frescoes, hallway)

The middle, the eastern portico, the throne hall, the water wheel and the bath complex which contained the largest group

It is considered one of the most photographic wall paintings in the world from the early Islamic period

The most important examples of early Islamic art and architecture, and his short life was listed UNESCO World Heritage in 9122 AD.

Saint Hilarion Monastery: This monastery, which is a unique architectural model, brought together a large number of people

Architectural Models The Tell Umm Amer site (Khirbet Umm al-Tut) is located in Nuseirat, $2.2 \mathrm{~km}$ away 
South of Gaza City, the monastery served as an important station at the crossroads of the House of Egypt and Palestine

Syria and Mesopotamia

\section{research aims:}

Provide a clear and complete picture of these sites.

Find out about the most important architectural elements in these sites.

\section{Research Methodology:}

I will adopt the descriptive analytical approach in gathering information related to the existing architectural elements

In these buildings.

\section{Keywords:}

Monastery, waterwheel, al fresco.

المقدمة: ترك لنا أجدادنا نر اثاً عريقاً من المباني المعمارية بتنوع البيئة والثقافة والتقاليد في سلسلة من التطورات الحضارية،

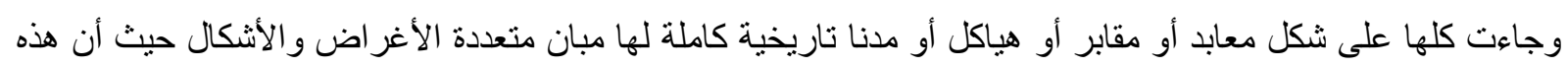

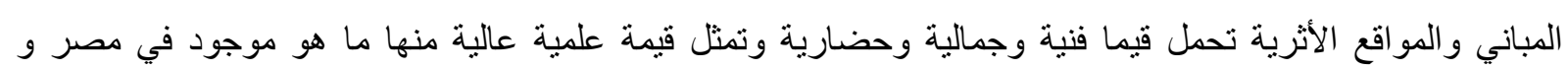
الأردن وفلسطين أيضاً. سوف أركز دراستي في هذه الدراسة على أهم المواقع و المباني الأثرية التي تحوي العديد من العناصر المعمارية كموقع دير القديس هيلاريون في فلسطين مدينة غزة أيضاً قصير عمرة في دولة النئة الأردن. أهداف البحث: تقديم صورة واضحة ومتكاملة عن هذه المو اقع. ـالوقوف على أهم العناصر المعمارية في هذه المو اقع. المصطات: الدير: مكان اختلاء الرهبان، و عادة ما يكون بعيد عن الزحمة وبه كنيس.

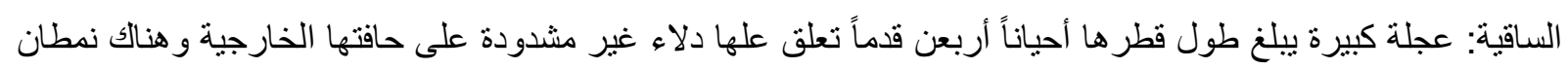
من السواقي إما عمودية أو أفقية. الفريسكو: تصوير جصي وهو احد أثكال الفن الني يتم من خلالها استخدام الجص كمادة لتمليط أو تجميص سقف أو جدران بشكل فني.

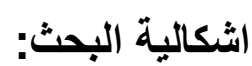
تتمثل في قلة المصادر و المراجع. منهج البحث: سأعتمد المنهج الوصفي التحليلي في جمع المعلومات المتعلقة في العناصر المعمارية الموجودة في هذه المباني. 

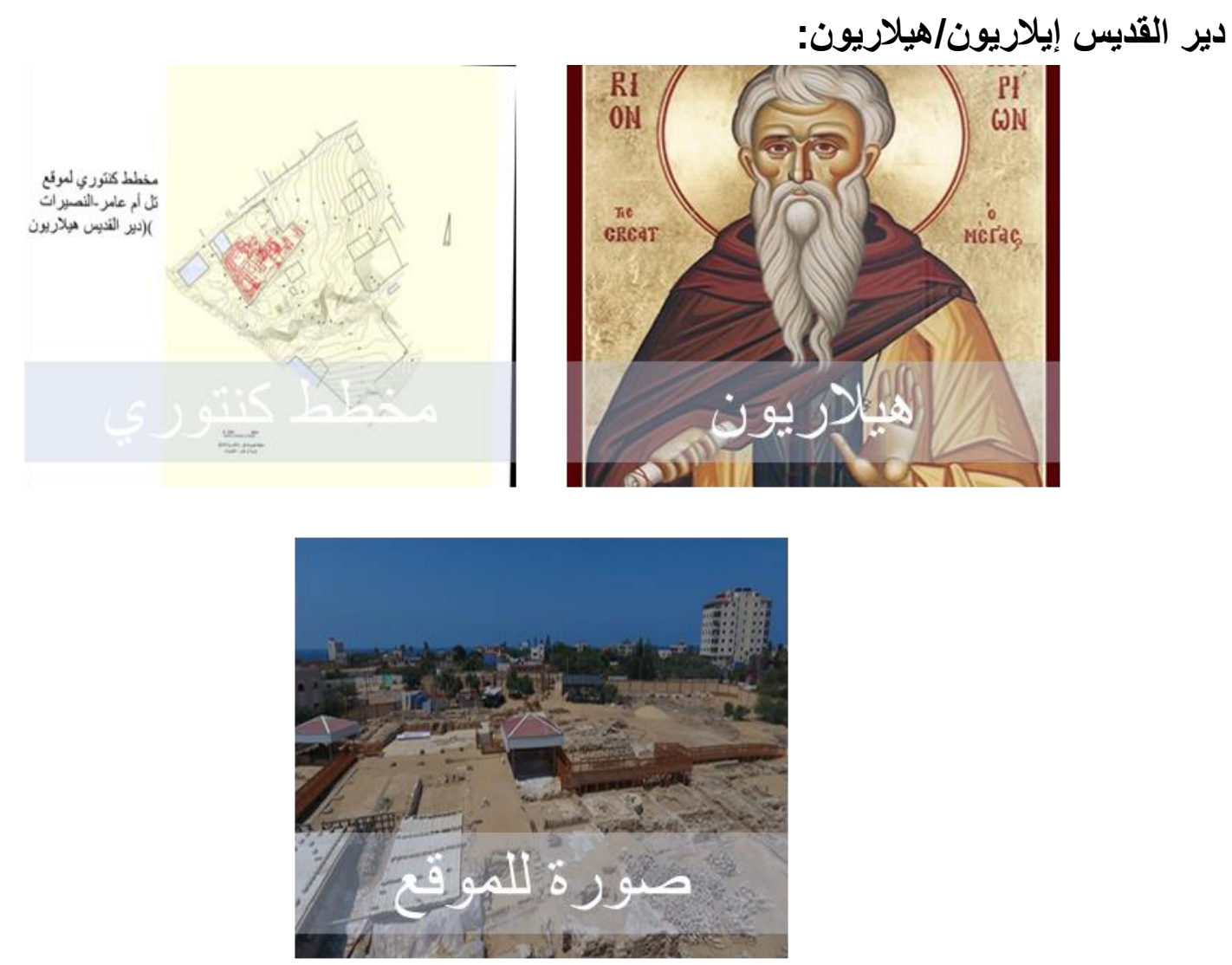

يعد من أكبر الأديرة وأقدمها في فلسطين وبلاد الثشام ،كانت تل أم عامر /خربة أم التوت في (طابثا) هي مسقط رأس

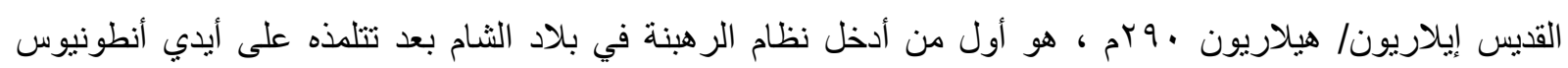

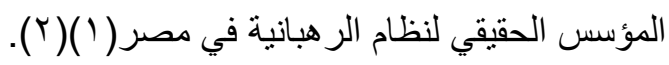

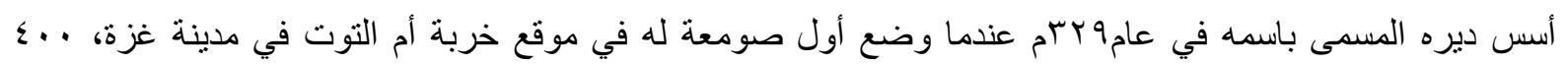

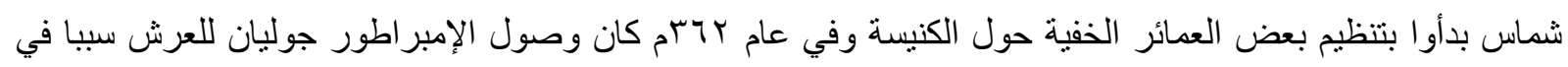

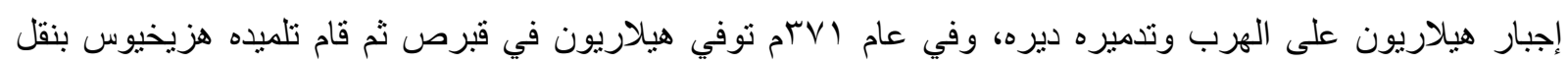

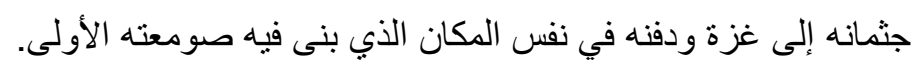

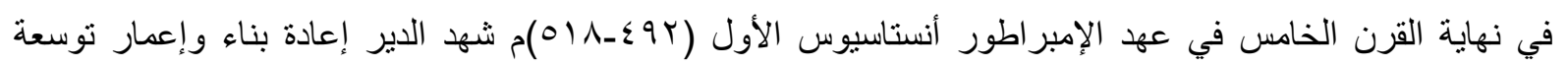
كنسية الدير لازدياد عدد المتعبدين ونم العثور على اسم الامبراطور إيلاريون مدون في نص تأسيسي على أرضية فسيفساء مذبح الكنيسة بعد التوسعة الثانية الذي جرى لهائ أما في القرن السادس حدث تطوير لكل منشآت الدير على الأغلب أن يكون الأسقف ماركيانوس قد ساهم بشكل كبير في

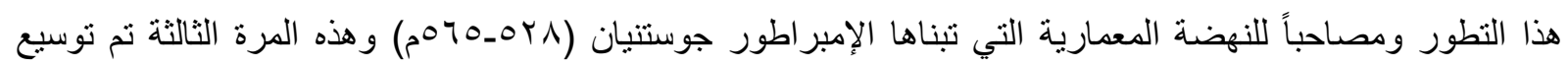
الكنيسة وهي التوسعة الموجودة حاليا المرتبطة مع قبو الديماس الكنسي الذي يحتوي على تابوت به رفات القديس هيلاريون..

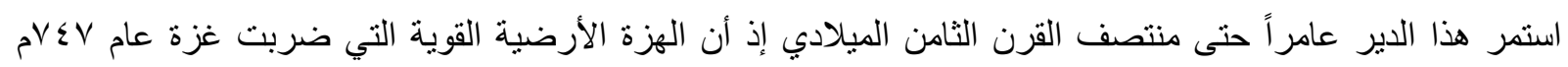

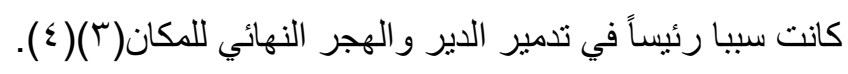


يحتوي موقع دير القديس هيلاريون على كنيستين وقبو الديماس الكنسي به تابوت عثر على جزء من غطاءه كان بداخله

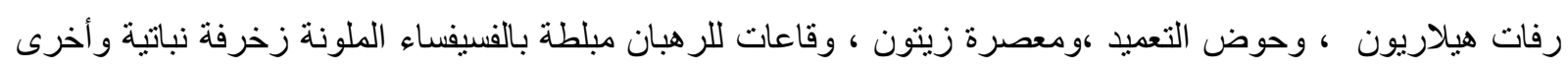

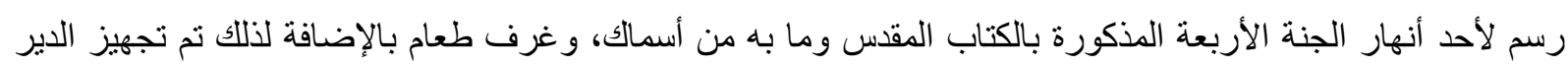
بحمامات تتكون من ثلاث غرف الساخنة و الفاترة و الباردة.

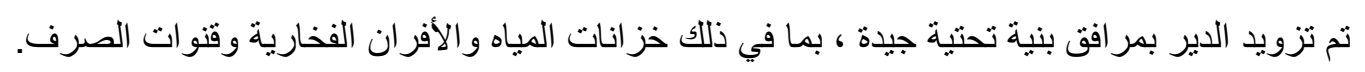

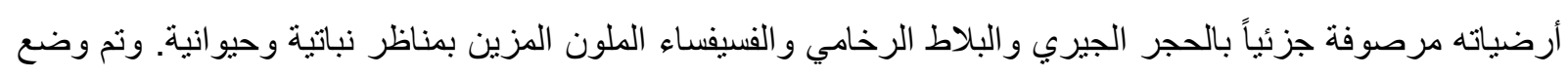

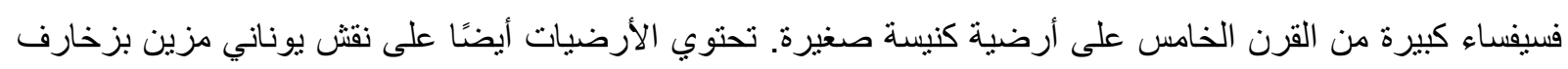

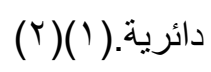

\section{بعض أرضيات الفسيفساء:}

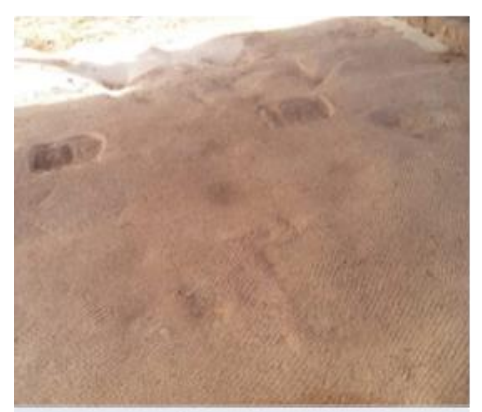

من العصر الأموي

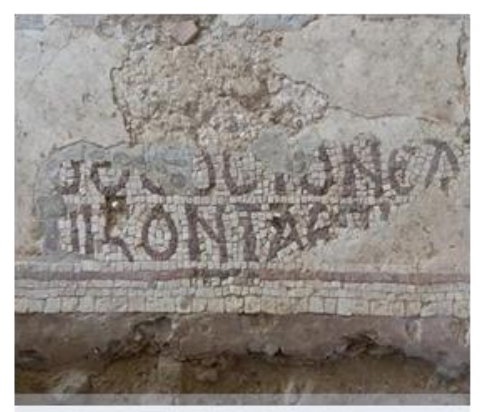

اسم هيلاريون

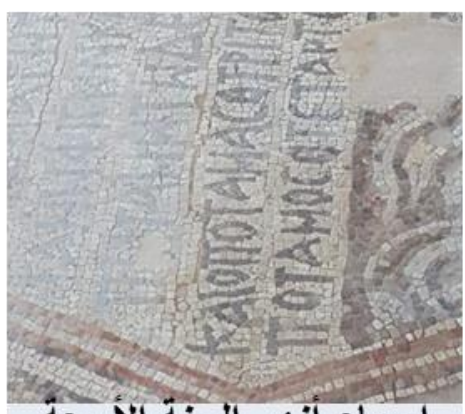

اسماء أنهز الجنة الاربعة باللغة النلاتينية

بعض التيجان والأعمدة التي عثر عليها في الموقع:
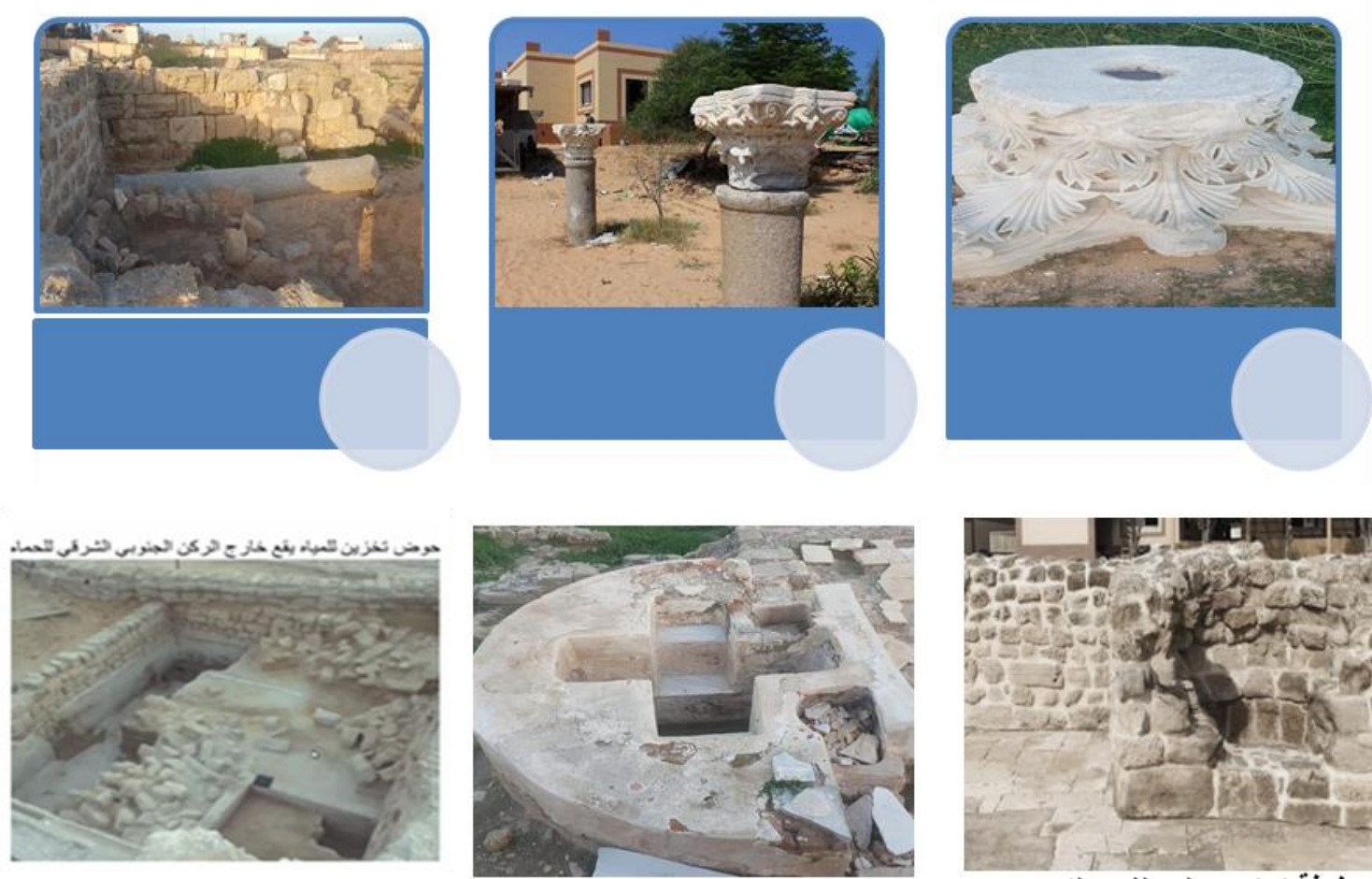

حوض العميد

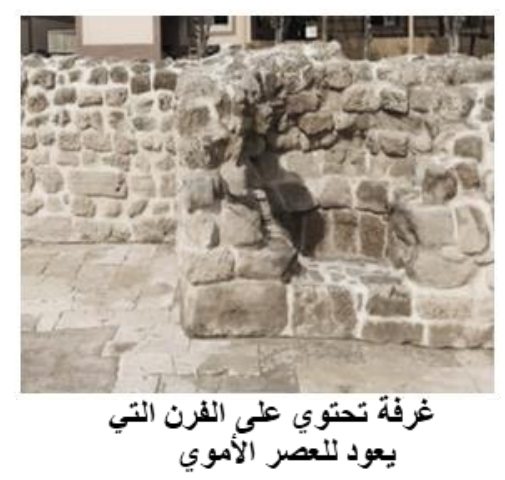




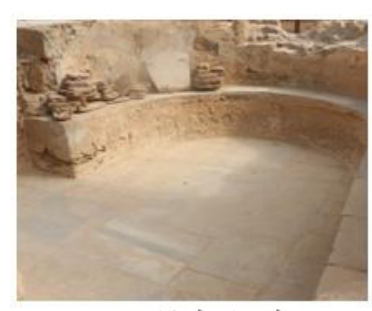

الحمام البخاري
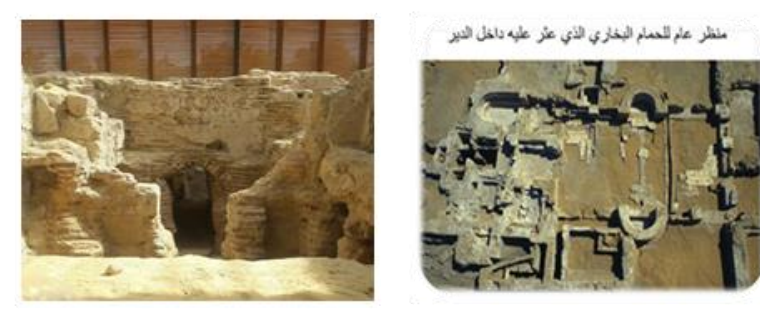

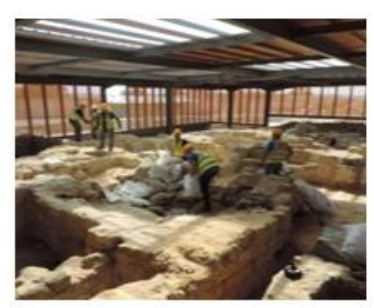

قصير عمرة:

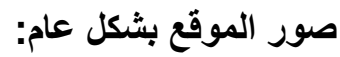
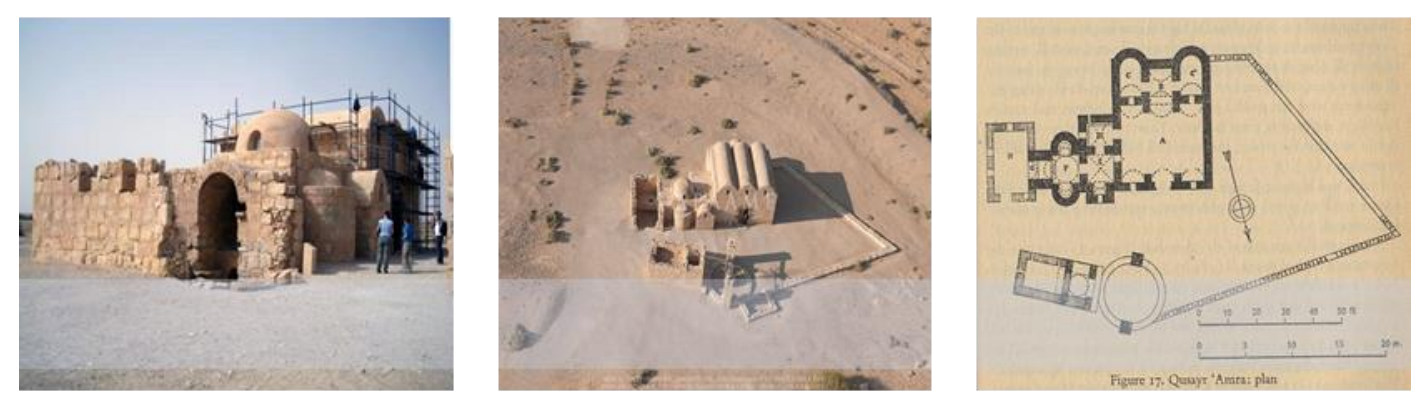

يقع القصر في شمال الصحر اء الأردنية في منطقة الأزرق حوالي • ال اكم شمال شرق العاصمة عمان.

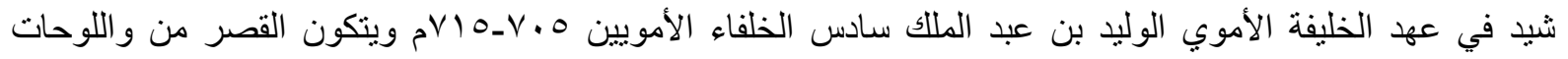

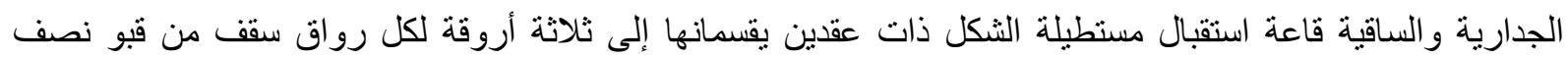
دائري ويتصل الرواق الأوسط من الجهة الجنوبية بحنية كبيرة على جانبيها غرفتان صغيرتان تطلان على حديقتين

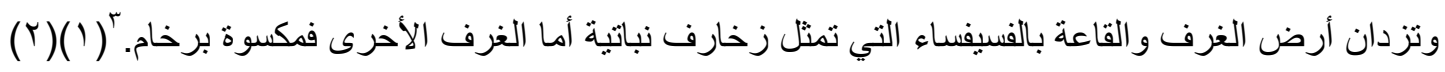

\section{قاعة الاستقبال وما يلحق بها من مبان:

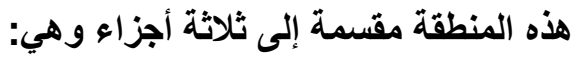
الصالة الرئيسة ومساحة مستطيلة أيضا تغطيها أقبية ثلاثة طويلة مرتكزة على دلى دعامات أربع و هذه الصالة تقضي إلى حنية العرش المسنطيلة الثكل و المغطاة بقبو نصف دائري منخفض على المستوى من سقف صالة الاستقبال. و هنالك صورة يعتقد أنها للخليفة الوليد بن عبد الملك تزين الدخلة التي بصدر هذه الحنية والقسم الثالث بهذه المنطقة هو مكان إقامة الخليفة والمكون من غرفتين مستطيلتين تقعان على جانبي حنية العرش هذه وشكل سققهما على قبو برميلي ونو افذها هاتين الغرفتين عبارة عن فتحات عالية تطل على صالة الاستقبال في جتهما الثمالية.( ( ) الحمام وملحقاته: الحمام يقع على يسار صالة الاستقبال وهو من ثلاث غرف رئيسية وهي القاعة الباردة و القاعة الدافئة والقاعة الساخنة و هذه الغرف مربعة الثكل ويغطيها قبو طولي بمحو عامودي على الصالة الاستقبال وتغطيها رسوم مائية و القاعة الدافئة ttipiarium

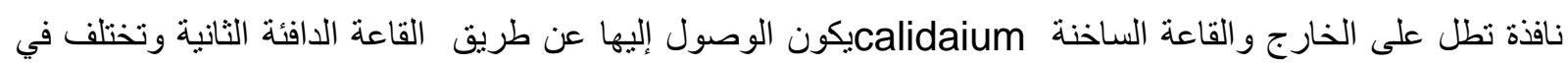
مسقطها وطريق تسقيفها فهي مكونة من مساحة مربعة مغطاة بقبة ضحلة مقامة على مثلثات كروية وفي هذه القاعة يوجد حوض مثمن رخامي يصل إليه الماء عن طريق أقصاب فخارية مخفية في باطن الأرض أنية من الموقل لتسخين المياه

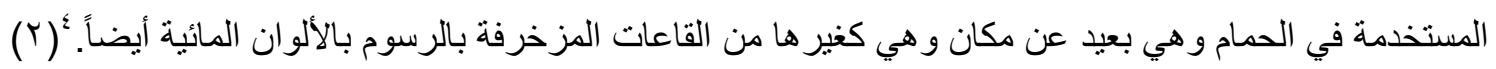


تنوعت بين رسوم صيد واستحمام ورسوم رمزية لآلهة الثعر و الفلسفة والنصر عند الإغريق وأخرى عن مراحل العمر المختلفة وبعض النجوم و إلى جانب كل هذا صورتان الأولى تمثل الخليفة الوليد بن عبد الملك على عرشه وحوله هالة وفوقه مظلة ترنكز على عمودين حلزونيين ويجف به شخصان وكتابة كوفية باللون الأبيض فوق خلفية زرقاء ( ( )و الثانية هي صورة كتب فوقها كلمة نيخ بمعنى النصر وهي ذات علاقة مباشرة بالصورة الثهيرة (أعداء افسلام) و التي تتألف من ستة أثخاص برتدون الملابس الفاخرة ثلاثة في المقدمة وثلاثة في المؤخرة وفوق أربعة منهم كتابة

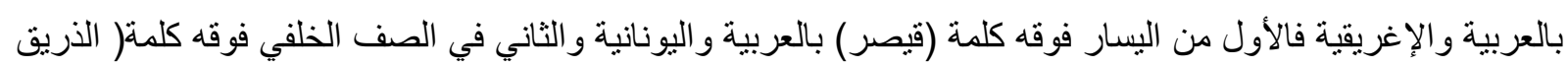
) آخر ملوك القوط في اسبانيا والثالث في الصف الأمامي فوق كلمة كسرى والرابع في الصف الخلفي فوقه كلمة ( النجانشي) والرسم يبين أن الأشخاص الذين في الصف الأول ملوك إمبر اطوريات كبيرة في حين رسم في الصف الخلفي

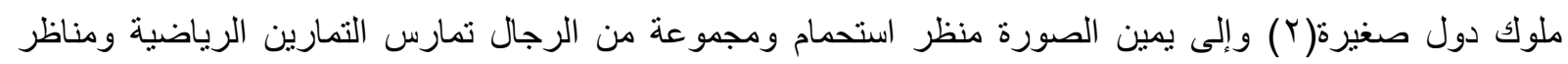
الصيد وبقايا صورتنين مع كلمتي تاريخ وفلسفة .(r). بالنسبة لرسومات الحمام زينت غرفة تغير الملابس بأثكال نباتية، وأثكال حيوانية كالدب الموسيقي، غزلان، طئن طيور مالك الحزين، ور اقصات، ورسوم لامر أة نصف مستلقية ثم امر أة تمسك بطفل و إلى يمين الباب تدخل امر أة أخرى و النساء تئهاء في الصورة عاريات أما غرفة الحمام الساخنة تعرض صور للأبراج فيوجد في الوسط الدب الأكبر والدب الأصغر

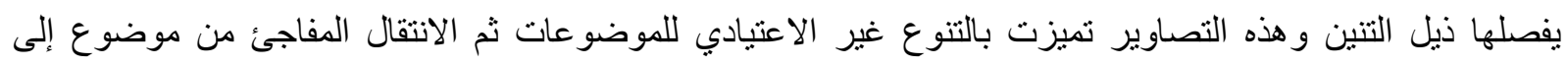
آخر(ء) و المؤثرات الفنية في هذا القصر تتوعت بين الأساليب الكلاسيكية والساسانية إلى جانب العناصر الثرقية المسيحية و هذه الرسومات داخل عناصر هندسية وتكررت داخل مربع أو معين في حركت منظمة لا متتاهية في شكل

هندسي وهذا الأسلوب يعطينا فكرة عن الصيغة المتميزة للفن الإسلامي.

الساقية:

تقع الى الثمال من المبنى الرئيسي ومحاذية له، نوجد منطقة دائرية لسير الحيو انات وبئر ماء بعمق • ب ـ • ع ، وخزان مياه حيث يرتفع منرين عن سطح الأرض لضمان الضغط الكافي لتدفق المياه عبر سلسلة من القنوات لتغذية مجمع من

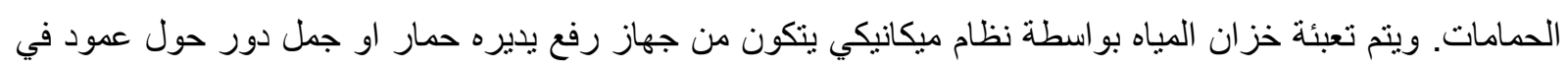

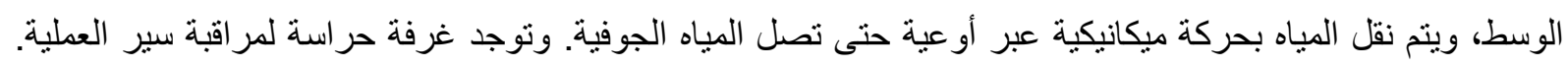

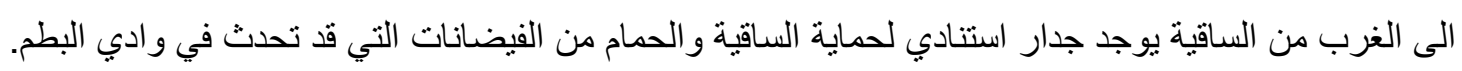

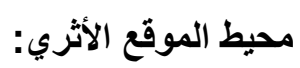
الساقية الثانية، القصر الرئيسي، برجية المر اقبة، السد المائي، المسجد، و مجموعة من البقايا الأثرية لا زالت تحت الدراسة منها الكهوف ومبنى شمال القصر( (') (r). ' ل 


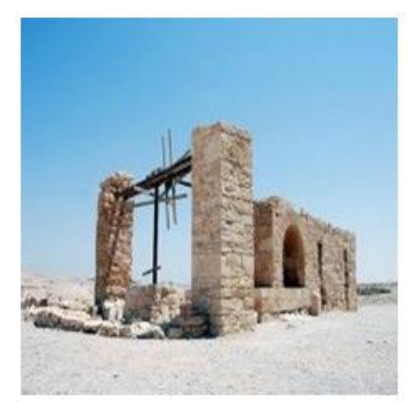

$$
\text { السماقية }
$$

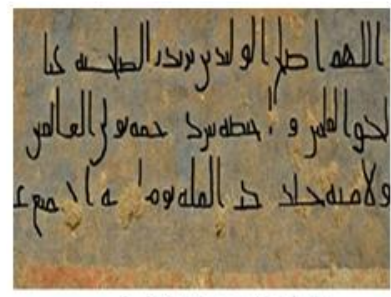

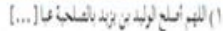
$\cos (x)$ (s)

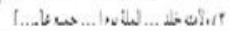

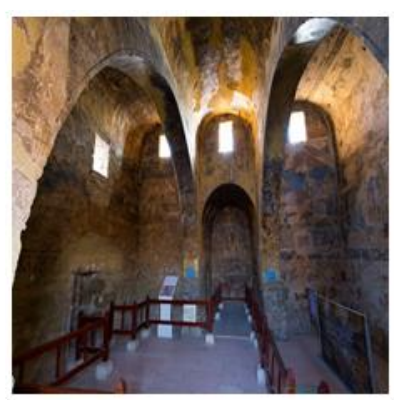

غرفة الاستقبال

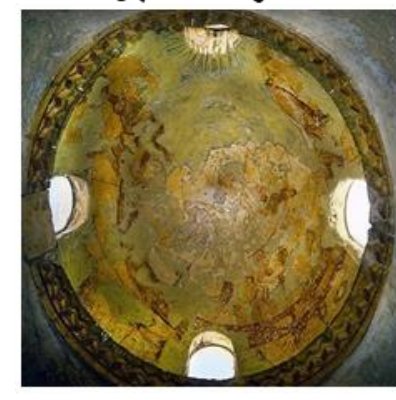

القبة الفلكية

بعض الجداريات قبل وبعد الترميم:

9Vا9 ام قام فريق إسباني بتغطية جميع الرسومات الموجودة في القصر بمادة الثلق وهي مادة صفراء مادة لحفظ

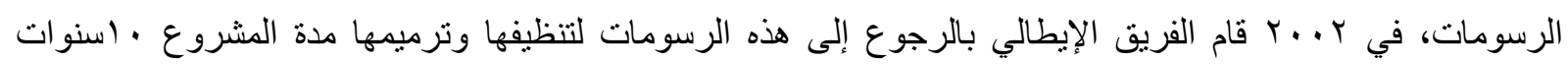
الهدف من المشروع هو إظهار الرسومات بشكلها الأصلي و إز الة مادة الثلق و إظهار ألوان الجداريات.

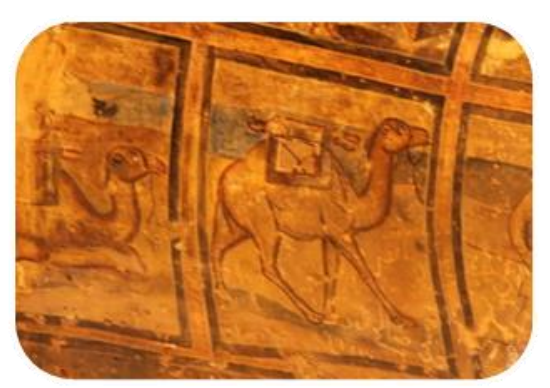

قبل التزميم

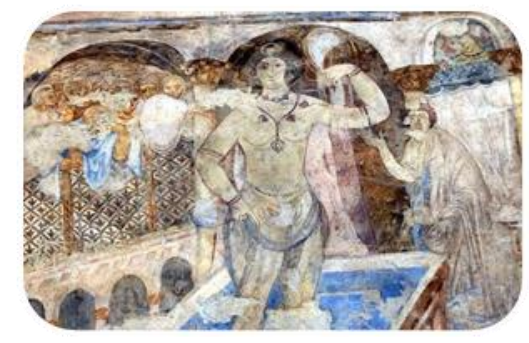

بعد الترميم

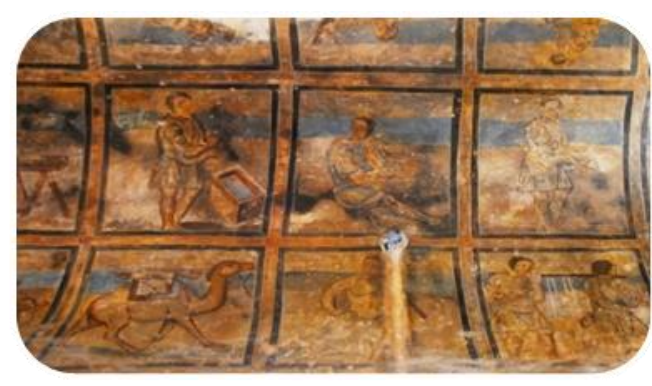

بعد التزميم

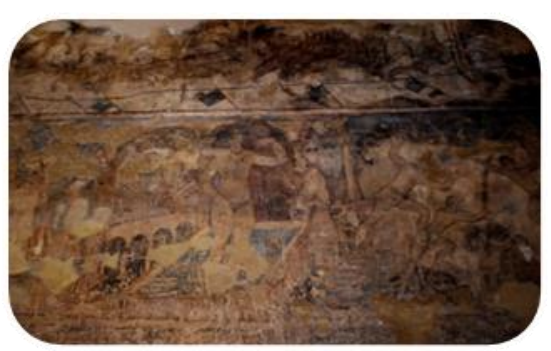

قبل التزميج 

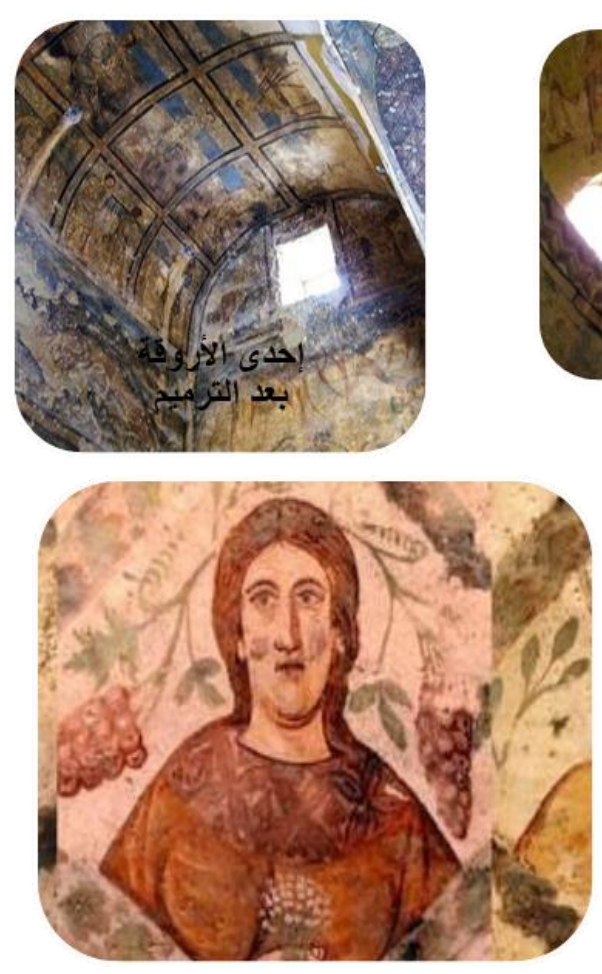

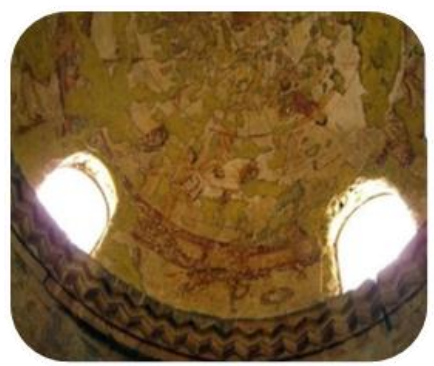

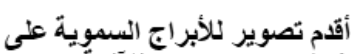
شكل كروي تصوير للالّلهة وصور للزهور

\section{إحدى النساء كمنظور إسلامي}

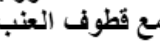

في صالة الاستقبال في الأعلى مشهذ صيد، وفي الأسفل من اليمين إلى اليسار مشهد الرياضة ومشهد المرأة اللغز ومشهر الملوك الستة.

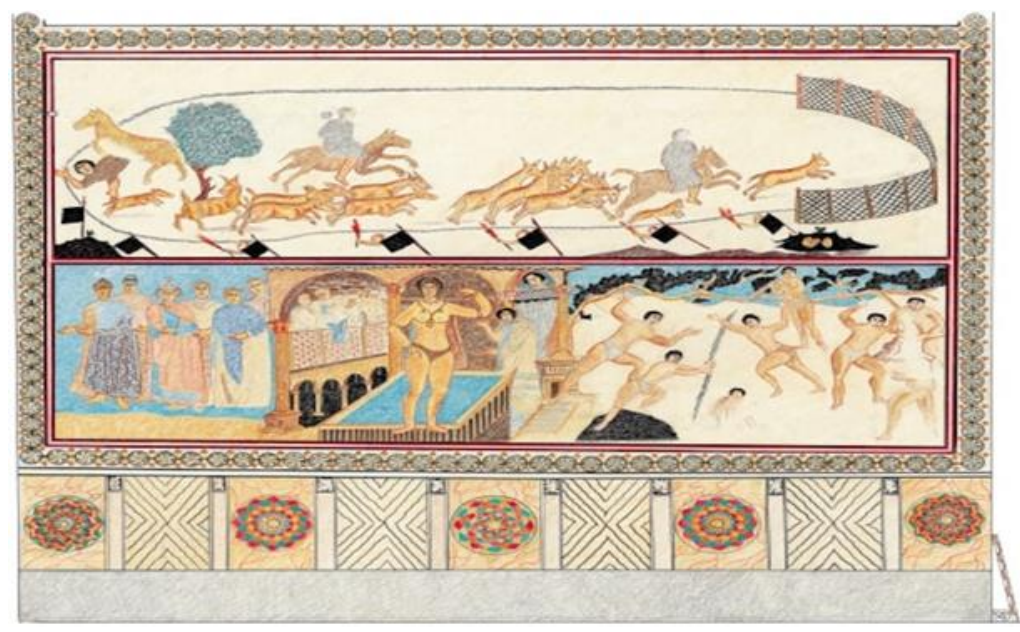

النتائج: هذفت هذه الدراسة إلى تسليط الضوء على أهم المواقع والمباني الأثرية ، بعد الاراسة السابقة يمكن الخروج بعدد من • يعتبر الاحتلال الصهيوني أحد أهم العوامل التي تمنع ترميم وصيانة المباني الأثرية و إدخال المواد لترميم المباني

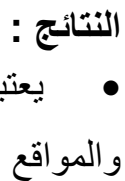
• • لا يوجد دليل كافي وشامل للمواقع الأثرية الموجودة في قطاع غزة.

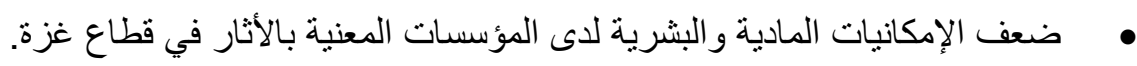
• عل التنقيات الأثرية في المباني المحيطة لقصير عمره. يجب دخول السو اح ضمن مراحل وذلك لضيق القصر من الداخل 
-تنفيذ أعمال الترميم من خلال مجمو عة عمل متفاهمة من خبر اء الفيزيائيين و الكيميائيين والأثريين و المهندسين المعماريين

وخبر اء صبانة.

-در اسة وفحص المنتجات التجارية المستخدمة في الصيانة و الترميم للوقوف على مدى ملاءمتها للمو اد الداخلة في مواد المبنى.

ـعدم الإفر اط في عمليات الترميم والاكتفاء بالقدر الضروري منها لضمان بقاء المبنى الأثري. ـ عدم القيام بأعمال الصيانة والترميم التي قد تؤدي إلى الإضرار أو إضعاف المواد الداخلة في تركيب المبنى الأثري.

توصيات ذات علاقة بالبحث العلمي في مجال الترميم والحفاظ على المباني والمواقع الأثرية:

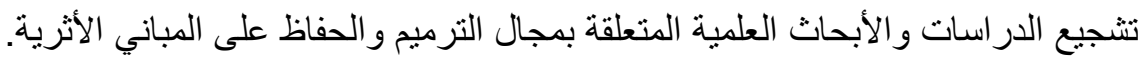

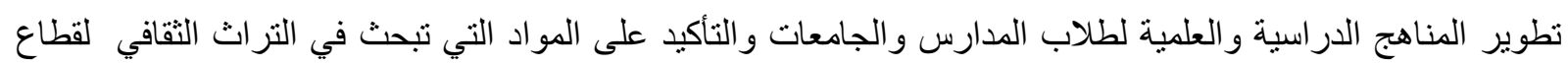
غزة وأساليب وطرق الحفاظ عليه.

توصيات ذات العلاقة بأهمية وأولوية الترميم:-

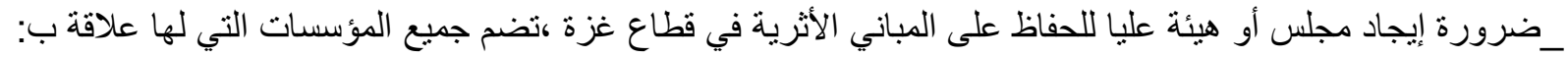
توثيق المباني و المو اقع الأثرية بشكل كامل وشامل و استخدام التقنيات الحديثة في التوثيق. إعداد دليل وطني شامل لعمليات الترميم الخاصة بالمباني الأثرية في قطاع غزة. _ـتكثيف الرقابة والإشراف من قلب الجهات المعنية ،على عمليات التنخل التي يقوم بها القائمون على المباني الأثرية

- $\quad$ 'ashraf kharishatan rayiys qism mawqie qasr eumratin.

• ثروت عكاشة، التصوير لإسلامي الديني والعربي، بيروت المؤسسة العربية لدراسات والنشر تاريخ الإصدار

- tharwat eakashat, taswir aldiyanat al'iislamiat walearabiat, muasasat bayrut alearabiat lildirasat walnashr, tarikh al'iisdar 1977.

$$
\text { جريدة الدستور الأردنية. }
$$

- $\quad$ sahifat aldustur al'urduniat.

- $\quad$ zayd ghawi, alsiyahat fi al'urdun

$$
\text { زيد غوي، السياحة في الأردن }
$$$$
\text { سليم المبيض، النصر انية وآثار ها في غزة وما حولها }
$$

- $\quad$ salim almubid almasihiat watharuha fi ghazat wama hawlaha سامي المغلوت، أطلس الأديان

- sami almaghlut, 'utlis al'adyan

$$
\text { عثمان الطباع، اتحاف الأعزة في ناريخ غزة، }
$$

- euthman altibae, 'athara aleizat fi tarikh ghazat. 
- kamirun kariaswil, eilm alathar al'iislamiat.

كاميرون كريزويل، الآثار الإسلامية . - ك

- muhamad wahayb alhikayat alshaebiat fi alzariqa' al'urduni

محمد وهيب، الحكاية الثعبية في الأردن الزرقاء.

- mustafaa aldabagh, watanuna filastin

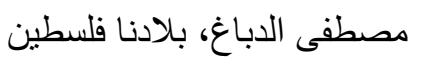

$$
\text { مروان أبو سويرح، الأرض والإنسان في الزوايدة }
$$

- marwan 'abu suayrah al'ard walrajul fi alzawayida

$$
\text { محمود فزغلي، التصوير الإسلامي، الدار المصرية اللبنانية، ا99 ام. }
$$

- mahmud fzghly taswir 'iislamiun albayt almisrii allubnanii $1991 \mathrm{~m}$.

- $\quad$ http://whc.unesco.org/en/tentativelists/5716

- /https://www.antiochpatriarchate.org/ar/page/813

$$
\begin{aligned}
& \text { ' (1) ' (1) مصطفى الدباغ، بلادنا فلسطين، صبسا }
\end{aligned}
$$

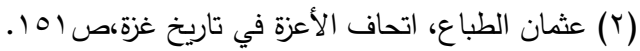

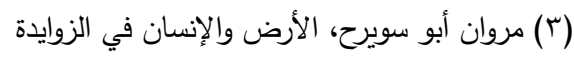

$$
\begin{aligned}
& \text { https://www.antiochpatriarchate.org/ar/page/813/ ( } 1 \text { ) }
\end{aligned}
$$

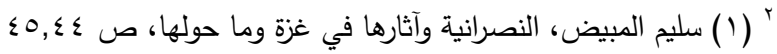

$$
\begin{aligned}
& \text { http://whc.unesco.org/en/tentativelists/5716(r }
\end{aligned}
$$

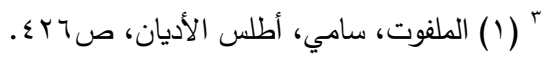

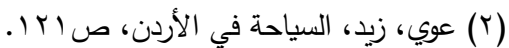

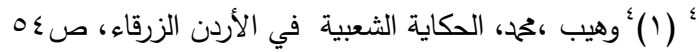

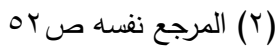

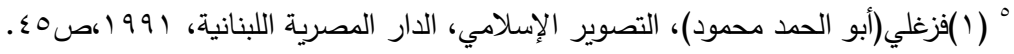

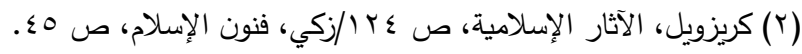

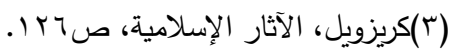

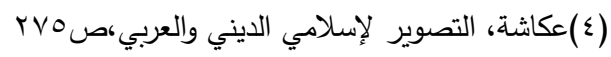

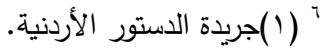

$$
\begin{aligned}
& \text { (ץ) أشرف خريشا، رئيس شعبة موقع قصر عمرة. }
\end{aligned}
$$

